\title{
Ethical conditions for accelerating COVID-19 vaccine research
}

\section{[version 1; peer review: 1 approved, 1 approved with}

\section{reservations]}

\author{
Maxwell J. Smith (D1) Ezekiel J. Emanuel2, Beatriz Thomé (D)3, Ross E.G. Upshur(D) \\ ${ }^{1}$ Faculty of Health Sciences, University of Western Ontario, London, Ontario, Canada \\ ${ }^{2}$ Department of Medical Ethics and Health Policy, University of Pennsylvania, Philadelphia, Pennsylvania, USA \\ ${ }^{3}$ Preventive Medicine Department, Federal University of São Paulo, São Paulo, Brazil \\ ${ }^{4}$ Dalla Lana School of Public Health, University of Toronto, Toronto, Ontario, Canada
}

V1 First published: 22 Oct 2020, 5:249

https://doi.org/10.12688/wellcomeopenres.16361.1

Latest published: 22 Oct 2020, 5:249

https://doi.org/10.12688/wellcomeopenres.16361.1

\begin{abstract}
There is a dire need to produce a safe and effective coronavirus disease 2019 (COVID-19) vaccine as quickly as possible to avert an enormous burden of morbidity and mortality. This need for speed has thus far resulted in significant deviations from standard approaches to vaccine research and regulatory approval. Yet, pandemics do not obviate the need for rigorous scientific evaluation of potential interventions and adherence to universal ethical standards. Consequently, steps taken to accelerate clinical research in the context of the current pandemic require an explicit ethical justification, particularly if we expect public trust in the vaccine. We argue that accelerating COVID-19 vaccine research can be ethically justified, but only if social value, scientific validity, and a favourable risk-benefit ratio can be maintained or enhanced when doing so.
\end{abstract}

Keywords

COVID-19, ethics, research, vaccine

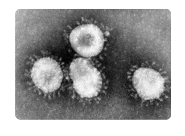

This article is included in the Coronavirus

(COVID-19) collection.

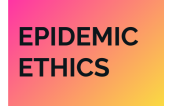

This article is included in the Epidemic Ethics:

Global issues in ethics and COVID-19 collection.

\section{Open Peer Review}

Approval Status

$?$

1

2

version 1

22 Oct 2020

?

1. Keymanthri Moodley (D), Stellenbosch

University, Cape Town, South Africa

2. Sonali Kochhar ID, Global Healthcare

Consulting, New Delhi, India

University of Washington, Seattle, USA

Any reports and responses or comments on the article can be found at the end of the article. 
Corresponding author: Maxwell J. Smith (maxwell.smith@uwo.ca)

Author roles: Smith MJ: Conceptualization, Investigation, Methodology, Writing - Original Draft Preparation, Writing - Review \& Editing; Emanuel EJ: Conceptualization, Investigation, Methodology, Writing - Original Draft Preparation, Writing - Review \& Editing; Thomé B:

Conceptualization, Investigation, Methodology, Writing - Original Draft Preparation, Writing - Review \& Editing; Upshur REG:

Conceptualization, Investigation, Methodology, Writing - Original Draft Preparation, Writing - Review \& Editing

Competing interests: No competing interests were disclosed.

Grant information: This work was supported by the Wellcome Trust through funding to the Public Health Emergency Preparedness and Response Ethics Network (PHEPREN) [221559]. PHEPREN funding is provided by The Wellcome Trust and the World Health Organization. This work was also supported by the Canadian Institutes of Health Research (C150-20 2019-11).

The funders had no role in study design, data collection and analysis, decision to publish, or preparation of the manuscript.

Copyright: (c) 2020 Smith MJ et al. This is an open access article distributed under the terms of the Creative Commons Attribution License , which permits unrestricted use, distribution, and reproduction in any medium, provided the original work is properly cited.

How to cite this article: Smith MJ, Emanuel EJ, Thomé B and Upshur REG. Ethical conditions for accelerating COVID-19 vaccine research [version 1; peer review: 1 approved, 1 approved with reservations] Wellcome Open Research 2020, 5:249 https://doi.org/10.12688/wellcomeopenres.16361.1

First published: 22 Oct 2020, 5:249 https://doi.org/10.12688/wellcomeopenres.16361.1 


\section{Disclaimer}

The views expressed in this article are those of the author(s). Publication in Wellcome Open Research does not imply endorsement by Wellcome.

\section{Introduction}

There is a dire need to produce a safe and effective coronavirus disease 2019 (COVID-19) vaccine as quickly as possible to avert an enormous burden of morbidity and mortality. This need for speed has thus far resulted in significant deviations from standard approaches to vaccine research and regulatory approval. For instance, Russia, China, and the United Arab Emirates have each authorized COVID-19 vaccination outside of clinicals trials ${ }^{1-3}$, and the US Food and Drug Administration has expressed its willingness to consider granting emergency authorization for a COVID-19 vaccine before phase three trials are completed ${ }^{4}$. In addition, the World Health Organization (WHO), via the COVAX pillar of its Access to COVID-19 Tools (ACT) Accelerator, is coordinating a global approach to the acceleration of COVID-19 vaccine $R \& D^{5}$. The WHO's protocol for its 'Solidary Vaccine Trial' calls for only some of its trial sites to perform detailed safety assessments in each treatment arm (as typically done in phase two trials), where other study sites will proceed directly to phase three testing following the review of early safety assessments of vaccine candidates ${ }^{6}$.

Accelerating the evaluation and development of vaccines is not without precedent. Some phase two enrolment began in the absence of published phase one data during Ebola vaccine trials ${ }^{7}$. In addition, given the large number of people affected by the 2013-2016 Ebola outbreak and a high case-fatality rate, a WHO advisory panel argued on both ethical and evidential grounds that it was permissible to use unregistered interventions that have shown promising results in laboratory and animal models as potential treatments or for prevention, provided that certain ethical and scientific criteria were met $^{8}$.

Despite the need for speed, pandemics do not obviate the need for rigorous scientific evaluation of potential interventions and adherence to universal ethical standards ${ }^{9}$. Consequently, steps taken to accelerate clinical research in the context of the current pandemic require an explicit ethical justification, particularly if we expect public trust in the vaccine. We argue that accelerating COVID-19 vaccine research can be ethically justified, but only if social value, scientific validity, and a favourable risk-benefit ratio can be maintained or enhanced when doing so.

\section{Social value}

For research involving human participants to be ethical, it must have social value ${ }^{10}$. It is clear that COVID-19 vaccine research has social value insofar as the development of a COVID-19 vaccine has the capacity to avert substantial morbidity and mortality worldwide.

But would social value be maintained or enhanced by deviating from the standard approach to clinical research and accelerating COVID-19 vaccine research? The virus is rapidly infecting the global population. Over one million people have already died. The more time that elapses between now and a vaccine, the more people will become ill and die. Moreover, no vaccine or therapy currently exists for this disease, making this research of even greater social value. Finally, the high social value of accelerated research in this context is symbolized by the fact that it responds to a threat for which a public health emergency of international concern has been declared $^{11}$. Given the substantial social value that a COVID-19 vaccine promises, there is an ethical imperative to proceed more rapidly.

However, social value may be diminished when studies are of low quality, research is unnecessarily duplicated, and data are not shared-concerns which may be particularly relevant for research conducted during pandemics ${ }^{12}$. Social value can be enhanced in this context via core protocols with adaptive designs, such as that proposed for the Solidarity Vaccine Trial, which promote collaboration and permit adding, dropping, and assessing multiple interventions under a common evaluative framework.

\section{Scientific validity}

Research must be conducted in a scientifically rigorous manner if it is to be ethical ${ }^{10}$. Consequently, even where the prospect of social value is high, any steps taken to accelerate COVID-19 vaccine research must be scientifically valid to avoid scientifically unreliable results.

Assessing the scientific validity of accelerating vaccine research by combining or skipping trial phases should be situated within a broader understanding of the reasons for a phased approach to clinical research. The phases of clinical research reflect a general schema that scientists follow to obtain valid and rigorous scientific data about the safety and effectiveness of investigational products. What is important is not the phases per se. They are merely a means to an end. Large-scale phase three testing has the capacity to generate sufficiently rigorous scientific safety and efficacy data. But proceeding with phase three testing in the absence of sufficient prior safety data has the potential to put participants at risk, and proceeding in the absence of sufficient efficacy data could be a waste of time and resources if it turns out the vaccine is not efficacious. Consequently, what is primarily at stake when accelerating research in this way isn't scientific validity, but rather a favourable risk-benefit ratio.

\section{Favourable risk-benefit ratio}

Research entails uncertainty about the degree of risks and benefits ${ }^{10}$. Typically, risks and benefits are balanced in clinical research by only increasing the number of individuals exposed to an investigational product as uncertainty is reduced regarding the product's risk-benefit ratio, as it generally does as we move through phases one, two, and three.

Consequently, the possibility exists that accelerating vaccine research would mean exposing a greater number of participants 
to an investigational product before a sufficient degree of certainty can be generated about the product's safety or efficacy. A justificatory condition for accelerating COVID-19 vaccine research is therefore the degree to which sufficient safety and efficacy signals are capable of being generated before large numbers of participants (or populations, in the case of regulatory approval) are exposed to the investigational product. So long as there is sufficient evidence of low risk and some chance of benefit in terms of potential protection against COVID-19, then accelerating the research approach would be justifiable.

To enhance the scientific rigour and ethical justification of this approach, several conditions should be fulfilled. Monitoring and oversight of safety by an independent data monitoring committee during all research phases should be enhanced. The data monitoring committee should review data sooner and more frequently and stopping rules should be stricter in order to quickly capture safety signals. Where possible, investigators should consider safety results from earlier phases when designing safety monitoring plans. The trial should not proceed in locales where this sort of monitoring is not possible. Finally, it will be important that phase three testing be followed up with robust post-trial surveillance that includes active monitoring for adverse effects, especially where phase three trials are not adequately powered to detect rare adverse events.

\section{Conclusion}

Finding ways to speed up research to produce a COVID-19 vaccine is an attractive prospect. Yet, the fact that we are in a pandemic does not provide sufficient warrant to lower our scientific or ethical standards ${ }^{12}$. Steps taken to accelerate COVID-19 vaccine research must therefore be subjected to rigorous scientific evaluation and adhere to universal ethical standards. This requires that steps taken to accelerate COVID-19 vaccine research maintain or enhance social value, scientific validity, and a favourable risk-benefit ratio. Any deviation from the standard approach to research should be explicitly justified in scientific and ethical terms, and this justification should be communicated transparently to researchers, review committees, research participants, and the public in order to engender trust in this research and the vaccine we hope it produces.

\section{Data availability}

Underlying data

No data are associated with this article.

\section{Acknowledgements}

A previous version of the manuscript is available from the Public Health Emergency Preparedness and Response Ethics Network (PHEPREN) website - https://media.tghn.org/articles/ Ethical_Conditions_for_Accelerating_COVID-19_Vaccine_ Research_-_PHEPREN_O710F4y.pdf
1. Jazeera A: Sputnik V: What we know about Russia's coronavirus vaccine. 2020.

Reference Source

2. Washington Post: China says it began public use of coronavirus vaccine a month ago, bypassing clinical trials. 2020.

Reference Source

3. The Wall Street Journal: In Global Covid-19 Vaccine Race, Chinese Shot Receives First Foreign Approval. 2020. Reference Source

4. Financial Times: FDA head says he is willing to fast-track Covid-19 vaccine. 2020.

Reference Source

5. World Health Organization: The Access to COVID-19 Tools (ACT) Accelerator. Reference Source

6. World Health Organization: An international randomised trial of candidate vaccines against CovID-19. Geneva Switzerland: World Health Organization, 2020.

Reference Source

7. Nason M: Statistics and logistics: Design of Ebola vaccine trials in West Africa. Clin Trials. 2016; 13(1): 87-91.

PubMed Abstract | Publisher Full Text
8. World Health Organization: Ethical considerations for use of unregistered interventions for Ebola virus disease: Report of an advisory panel to WHO. Geneva, Switzerland: World Health Organization. 2014. Reference Source

9. National Academies of Sciences, Engineering, and Medicine: Integrating clinical research into epidemic response: the Ebola experience. Washington, DC: The National Academies Press. 2017. Publisher Full Text

10. Emanuel EJ, Wendler D, Killen J, et al.: What makes clinical research in developing countries ethical? The benchmarks of ethical research. J Infect Dis. 2004; 189(5): 930-937. PubMed Abstract | Publisher Full Text

11. World Health Organization: Statement on the second meeting of the International Health Regulations (2005) Emergency Committee regarding the outbreak of novel coronavirus (2019-nCoV). Geneva, Switzerland: World Health Organization. 2020. Reference Source

12. London AJ, Kimmelman J: Against pandemic research exceptionalism. Science. 2020; 368(6490): 476-477. PubMed Abstract | Publisher Full Text 


\section{Open Peer Review}

\section{Current Peer Review Status:}

\section{Version 1}

Reviewer Report 10 February 2021

https://doi.org/10.21956/wellcomeopenres.17994.r42214

(C) 2021 Kochhar S. This is an open access peer review report distributed under the terms of the Creative Commons Attribution License, which permits unrestricted use, distribution, and reproduction in any medium, provided the original work is properly cited.

\section{Sonali Kochhar}

1 Global Healthcare Consulting, New Delhi, New Delhi, India

${ }^{2}$ Clinical Associate Professor. Department of Global Health, University of Washington, Seattle, USA

The letter speaks about the urgent need to develop and deploy a safe and effective COVID-19 vaccine as rapidly as possible to avert the tremendous morbidity and mortality burden due to the pandemic. The urgency does not obviate the need for rigorous scientific evaluation of the vaccines during the clinical research and adherence to the ethical standards. Accelerating COVID-19 vaccine research can be ethically justified if social value, scientific validity, and a favourable risk-benefit ratio can be maintained or when doing so. Social value can be enhanced via core protocols with adaptive designs e.g. as proposed for the Solidarity Vaccine Trial, which promote collaboration and permit adding, dropping, and assessing multiple interventions under a common evaluative framework. Phase three trials should be done only when there is sufficient prior safety and immunogenicity data to support the trial conduct. The monitoring by an independent data monitoring committee should be enhanced to closely monitor the safety of the trial participants during all research phases. The data monitoring committee should review data sooner and more frequently and stopping rules should be stricter in order to quickly capture safety signals. The trial should be conducted in locales where this sort of monitoring is possible. It is important that phase three testing be followed up with robust post-trial surveillance including for active monitoring for adverse effects, especially as the phase three trials are not adequately powered to detect rare AEs.

\section{Is the rationale for the Open Letter provided in sufficient detail?}

Yes

Does the article adequately reference differing views and opinions? Yes

Are all factual statements correct, and are statements and arguments made adequately supported by citations?

Yes 


\section{Is the Open Letter written in accessible language? \\ Yes}

Where applicable, are recommendations and next steps explained clearly for others to follow?

Yes

Competing Interests: No competing interests were disclosed.

Reviewer Expertise: Clinical research for vaccines, ethical considerations, safety surveillance, Pharmacovigilance, introduction of new vaccines

I confirm that I have read this submission and believe that I have an appropriate level of expertise to confirm that it is of an acceptable scientific standard.

Reviewer Report 08 December 2020

https://doi.org/10.21956/wellcomeopenres.17994.r41236

(c) 2020 Moodley K. This is an open access peer review report distributed under the terms of the Creative Commons Attribution License, which permits unrestricted use, distribution, and reproduction in any medium, provided the original work is properly cited.

\section{Keymanthri Moodley}

Centre for Medical Ethics and Law, Department of Medicine, Faculty of Medicine and Health Sciences, Stellenbosch University, Cape Town, South Africa

The need for rigorous conduct and robust regulatory review of COVID-19 vaccine research is highlighted based on three criteria of a pre-pandemic research ethics framework. There is no explanation for why these criteria were selected and why other criteria are less important informed consent, community engagement, fair subject selection - despite the original framework requiring all criteria to be met to make scientific research ethical. The Emanuel framework is typically acknowledged as a pre-pandemic framework for research ethics review. A public health ethics approach to research during a public health crisis is not discussed.

If more frequent DSMB monitoring is necessary to address the safety concerns of vaccine trials during the pandemic, does this mean that LMIC settings that cannot abide by the need for more frequent monitoring should be excluded from conducting vaccine trials during a public health emergency? What level of bias does this recommendation introduce and how does it impact on generalizability of findings from resource rich settings?

Alternate study designs such as Human Challenge Studies are not addressed.

While necessary and important, this letter provides insufficient guidance for Research Ethics Committees (RECs), regulatory authorities and researchers alike. 
Is the rationale for the Open Letter provided in sufficient detail?

Yes

Does the article adequately reference differing views and opinions?

No

Are all factual statements correct, and are statements and arguments made adequately supported by citations?

Partly

Is the Open Letter written in accessible language?

Yes

Where applicable, are recommendations and next steps explained clearly for others to follow?

No

Competing Interests: No competing interests were disclosed.

I confirm that I have read this submission and believe that I have an appropriate level of expertise to confirm that it is of an acceptable scientific standard, however I have significant reservations, as outlined above. 